\title{
Orofacial myofunctional characteristics of oral and oronasal breathers
}

Fabíola Maria Gonçalves Félix de Mattos ${ }^{(1)}$

(1) Universidade Estadual de Campinas UNICAMP, Campinas, São Paulo, Brasil.

Conflict of interests: Nonexistent

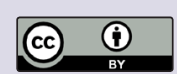

Received on: April 9, 2018

Approved on: June 6, 2018

Corresponding author:

Fabíola Maria Gonçalves Félix de Mattos Avenida Otacílio Tomanik, 1054 Apt $^{0} 24$ Jardim Bonfiglioli

CEP: 05363-101, São Paulo, São Paulo, Brasil

E-mail: fagonfelix@hotmail.com

\section{ABSTRACT}

Purpose: to compare the variables of the Orofacial Evaluation with Scores Protocol between nasal, oral and oronasal breathers.

Methods: a sample consisting of three distinct groups subdivided into nasal, oral and oronasal breathers, both genders, with ages ranging from 6 to 12 years. The characteristics of the orofacial musculature were measured using the protocol.

Results: there was a significant difference between oral and oronasal breathers compared to nasal ones $(p=0.0007)$ in specific variables such as palatal conformation ( $p=0.0073)$; mobility of tongue ( $p$ : 0.0111$)$ and lip $(p=0.0451)$, with a significant difference between the groups evaluated, also observed for the total protocol score $(p=0.0007)$ and posture ( $p: 0.0073)$, where the nasal respirators differ from the oral and oronasal ones, the latter being similar. As for lip $(p=0.0451)$ and tongue $(p=0.0111)$ mobility, the groups studied presented different results among themselves.

Conclusion: when comparing the groups of oral and oronasal breathers, in relation to the variables of the AMIOFE protocol, no statistically significant difference was seen between these two groups, which allows the conclusion that the orofacial myofunctional behavior between oral and oronasal breathers in this research, was similar.

Keywords: Speech, Language and Hearing Sciences; Breathing; Stomatognathic System 


\section{INTRODUCTION}

The consequences of inefficient breathing through the nose are already well known and described in the literature, both from the point of view of the general health of the organism and the compromises to the stomatognathic system (SS).

Therefore, keeping the lips occluded allows the child not only a good health condition but also the maintenance of the negative intra-oral pressure ${ }^{1}$ that is fundamental for the correct development of the bucofacial and neuromuscular, favoring the skeletal growth and the maintenance of the muscular tonic ${ }^{2-4}$.

Studies in the literature already report that in oronasal respirators the negative intra-oral pressure is also ruptured and causes compromises in the stomatognathic system ${ }^{3}$, due to the postural condition of the phonoarticulatory organs.

So, in the oronasal respirator, also called vicious ${ }^{5,6}$, mixed $^{7}$ or partial ${ }^{8,9}$, whether through habit or muscle memory, the mouth remains ajar, suggesting that the child who breathes sometimes through the mouth or through the nose ${ }^{10}$ may present oromyofunctional characteristics similar to those found in the oral respirator.

It is up to the speech-language pathologist to perform the assessment of the stomatognathic system in specific cases of orofacial myofunctional disorder ${ }^{11}$, that is "any alteration involving the oral and/or facial musculature that interferes with the growth, development and function of craniofacial structures". Oral Motricity of the Brazilian Speech and Hearing Society, 2/2003.

Thus, careful evaluation of the SS and its functions is the first step towards suitable therapeutic management, and accordingly, the quantification of the evaluation data tends to contribute to a greater credibility of the diagnosis and the results obtained.

Renowned authors in the area of orofacial motricity emphasize the importance of orofacial myofunctional evaluation and define strategies for its accomplishment, addressing morphology and stomatognathic functions, or both ${ }^{12}$.

Recognizing that the technical complexity of fine procedures and the restricted access to certain special materials make it difficult to carry out certain evaluations $^{13}$, which often do not replace clinical examination; a proposal for an essentially clinical protocol is of great value.

So, the authors Felício and Ferreira developed and validated a protocol with numerical scales (Orofacial
Evaluation Protocol with AMIOFE), to be used by speech therapists so that the perception of the examiner regarding the characteristics and orofacial behaviors observed can be expressed by numerical scales, allowing the measurement of the clinician's impression without the use of special materials.

The AMIOFE scales were developed at an interval of measurement, which does not imply a comparison between stimuli, or in the present case of orofacial conditions and behaviors, in order to assign the score ${ }^{13}$.

Using the interval scale, a number must be assigned within an established limit. Thus, the numbers or scores of the AMIOFE protocol were assigned, respecting the predetermined rule, that myofunctional orofacial conditions and normal orofacial behaviors would receive the highest scores and deviations from normality, altered conditions or disabilities in behavior would receive scores gradually lower, according to the examiner's perception ${ }^{13}$.

The AMIOFE protocol was used in its entirety in the research participants, with the main goal of classifying the oronasal and oral respiratory modes, being the mild oronasal respiratory mode considered oronasal and the severe oronasal or oral ${ }^{14}$, in addition to all participants receiving values that corresponded to their myofunctional orofacial condition.

The AMIOFE points to the $25 \%$ percentile in the analysis of Sensitivity, Specificity, Positive and Negative Predictive Values, and Prevalence of Orofacial-DMO Myofunctional Disorder, being considered as having BMD, subjects with a score equal to or less than $80^{15}$.

The aim of the present study was to compare the variables of the AMIOFE protocol between oral (OR) and oronasal respirators (RON), in order to observe if there are similarities between the groups regarding the appearance and posture of the lips, tongue, face, cheeks and palate; mobility of the lips, tongue, jaw and cheeks, and in the neuro-vegetative functions of respiration, chewing and swallowing.

\section{METHODS}

The study was approved by the Research Ethics Committee of the State University of Campinas (UNICAMP), under no.1.125.115 according to Resolution 466/12 of the National Health Council (CNS). The data of this article are an integral part of the dissertation entitled "The impact of oronasal breathing in relation to the perioral musculature" and the legal guardians of the selected patients signed the Free and 
Informed Consent Term (TCLE), in order to authorize data collection.

\section{Sample}

It consisted of 48 patients, divided equally into three groups, Nasal Respirators (RN), Oral Respirators (RO) and Oronasal Respirators (RON), aged between 6 and 12 years old, males and females, selected from the waiting list of a Basic Health Unit. The selected groups for this article were RON and RO.

Inclusion criteria: patients with typical development, with ages ranging from 6 to 12 years old and complete chart with otorhinolaryngological evaluation of the upper airways and speech-language pathology in the respiratory mode.

With the application of AMIOFE, the groups were classified into:

- ORAL RESPIRATOR (RO): obstruction of the upper airways, difficulty breathing through the nose, showing signs of fatigue, dyspnea and needing to open the mouth to inspire, rest and chewing ${ }^{11}$.

- ORONASAL RESPIRATOR (RON): clearing the upper airways, breathing through the mouth and nose, but being able to breathe through the nose without showing signs of fatigue or dyspnea ${ }^{11}$.

Exclusion criteria: neurological, cognitive impairment, peripheral and/or central facial paralysis, syndromes, cleft lip and palate, making use of myorelaxing medicine, facial trauma, submitted to myotherapic and/or orthodontic and/or facial orthopedic treatment.

\section{Procedures}

The evaluation consisted of the analysis of the charts for investigation of otorhinolaryngological opinion regarding upper airway obstruction and the application of the Orofacial Evaluation with Orofacial Scores Protocol (AMIOFE) ${ }^{11}$. AMIOFE has also been used to define the respiratory mode ${ }^{11,14}$ and is applied integrally, since for the definition of the different respiratory modes it is necessary to observe the patient throughout the evaluation. This article will present the findings of the clinical orofacial myofunctional evaluation.

According to the protocol, the sum of the scores ranges from 0 to 103 points, and the higher the value, the better the myofunctional orofacial condition ${ }^{7}$. Being considered with myofunctional disorders those with percentage less than $25 \%$ of the total value or score below 80 points $^{15}$.

For the application of AMIOFE it was necessary to use the following materials: tripod for fixing the SONY digital camera, model DSC-W130, Super Steady Shot, digital timer, two chairs with backrest for the examiner and patient, respectively, disposable gloves, spatulas wood, disposable cups, room temperature mineral water and $\mathrm{BONO} \otimes$ chocolate biscuit.

To determine the groups in this study, the mild oronasal respirator was considered to be an oronasal respirator and a severe oronasal respirator as an oral respirator ${ }^{14}$. Regarding gender, the Oral breathing Group (GO) was composed of six girls and ten boys and the Group with Oronasal breathing (GON) by four girls and twelve boys. The subjects were assessed individually, remaining seated in a reclining chair with their feet flat on the floor. The evaluation of the stomatognathic system included: appearance of face, palate, postural condition/position of lips, mandible, cheeks and tongue; mobility of the lips, tongue, cheeks and jaw and performance in chewing and swallowing functions of liquid and solid cake.

To verify the appearance of the face was considered symmetry between the right and left sides, mild or severe asymmetry. For the appearance of the palate it was considered normal, narrowed width (narrow/ severe). For the postural condition of the lips, it was considered: normal posture, occlusion of the teeth without free functional space, open mouth with mild and excessive dysfunction and opening of the mouth with severe dysfunction. The vertical posture of the mandible was classified as: normal posture, occlusion of the teeth without free functional space, excessive mouth opening with severe dysfunction. For cheek appearance, the criteria were: normal, increased volume or flaccid/arched mild or severe. For the position of the tongue it was observed whether it was contained in the oral cavity, interposed to the dental arches with adaptation or dysfunction and interposed to the dental arches with excessive protrusion.

In order to evaluate the mobility of the stomatognathic system, the movements of the lips (protrusion, protrusion, lateroprotrusion on the right and left) of the tongue were requested (protrusion, lateralization to the right, lateralization to the left, lifting, lowering and ability to maintain the tongue protruding for five seconds), cheeks (inflate, suck, retract and lateralize) and the mandible (protrusion, lowering, elevation, lateralization to the right and left). 
In the analysis, they were considered normal: isolated movements of each component, with precision and without tremor. The following were considered as changes: lack of precision in movement, tremor, associated movements of other components (for example, lips that accompany the movements of the tongue), and the inability to perform the movement. The examiner assigned scores on a point scale, according to AMIOFE, where: $3=$ normal, $2=$ insufficient ability and 1 = lack of ability or non-achievement of the task. Regarding mandibular movements, extension measures and symmetry/asymmetry were also considered during opening and closing, oral right and left laterality and protrusion. At swallowing, it was considered normal pattern when the subject presented tongue contained in the oral cavity, contraction of the lifting muscles and the anterior seal of the oral cavity without effort.

In the mastication, the subject was instructed to chew a Bono ${ }^{\circledR}$ stuffed biscuit in a habitual way, being observed the grinding, the presence of associated body movements and if there was food escape. The following aspects were considered: trituration: alternating bilateral, simultaneous bilateral, chronic unilateral (95\% of the time on the same side of the oral cavity), masticatory preference unilateral $(66 \%$ of the same side), or previous; and total time for food consumption. The chronometer was always activated after the food was placed in the oral cavity and the beginning of the grinding; it was stopped when the final swallowing of each portion occurred. Subsequently, according to the protocol, scores were assigned.

To describe the profile of the sample according to the variables under study, tables of the descriptive statistics of the numerical variables with values of mean, standard deviation, minimum and maximum and median values were made. The tests used were Chi-Square, Fisher and ANOVA. The Mann-Whitney test was used to compare the variables. The level of significance adopted for this study was $5 \%$.

\section{RESULTS}

\section{Characterization of the sample}

There were no significant differences between the groups, according to Table 1.

Table 1. Sampling in relation to gender in different respiratory patterns

\begin{tabular}{|c|c|c|c|c|}
\hline \multirow{2}{*}{ Gender } & \multicolumn{3}{|c|}{ Groups } & \multirow{2}{*}{ Total } \\
\hline & $\mathrm{RN}$ & R0 & RON & \\
\hline Female (freq.) & 5 & 6 & 4 & 15 \\
\hline$\%$ & 31.25 & 37.50 & 25 & \\
\hline Male (freq.) & 11 & 10 & 12 & 33 \\
\hline$\%$ & 68.75 & 62.50 & 75.00 & \\
\hline TOTAL & 16 & 16 & 16 & 48 \\
\hline
\end{tabular}

freq.: frequency, \%: percentage, RON = Oronasal Respirator p: 0.7476 (Chi-Square and Fisher, $p<0.05$ ), frequency:\%, RN $=$ Nasal Respirator, OR $=($ Chi-Square and Fisher, $p<0.05)$.

Regarding age, the data also showed no significant difference between the groups, according to Table 2 .

Table 2. Sampling in relation to age

\begin{tabular}{ccccccc}
\hline Group & N & Mean & Median & D.P & Minimum & Maximum \\
\hline RN & 16 & 7.94 & 7.5 & 2.05 & 6.0 & 12 \\
R0 & 16 & 6.69 & 6.0 & 1.01 & 6.0 & 9 \\
RON & 16 & 7.00 & 6.0 & 1.37 & 6.0 & 10 \\
\hline
\end{tabular}

N: sample, D.P: Standard Deviation, RN = Nasal Respirator, RO = Oral Respirator, RON = Oronasal Respirator, p: 0.1550 (ANOVA test, $p<0.05)$ 


\section{Results of Orofacial Evaluation with scores Protocol - AMIOFE:}

The findings between GN, GO and GON, regarding the values of the maximum and minimum scores; mean and standard deviation for AMIOFE are set in Table 3 below. It was observed that the RON and RO groups had total scores lower than 80 , therefore with a diagnosis of Orofacial Myofunctional Disorder (BMD) ${ }^{16}$.

Table 3. Mean AMIOFE results in the groups studied. GN: respirator group nasal, GO: oral respirator group, GON: oronasal respirator group

\begin{tabular}{cccccccc}
\hline Group & Variable & N & Mean & Median & D.P. & Minimum & Maximum \\
\hline GN & Age & 16 & 7.94 & 7.50 & 2.05 & 6.00 & 12.00 \\
& AMIOFE & 16 & 85.81 & 85.50 & 6.16 & 75.00 & 94.00 \\
GO & Age & 16 & 6.69 & 6.00 & 1.01 & 6.00 & 9.00 \\
& AMIOFE & 16 & 77.81 & 77.50 & 6.21 & 69.00 & 89.00 \\
GON & Age & 16 & 7.00 & 6.00 & 1.37 & 6.00 & 10.00 \\
& AMIOFE & 16 & 77.56 & 78.50 & 6.83 & 60.00 & 86.00 \\
\hline
\end{tabular}

With the variables: age and AMIOFE score; $\mathrm{N}$ (sample number); values of mean, median, D.P: standard deviation and maximum and minimum values found. (Chi-Square and Fisher, $\mathrm{p}<0.05)$.

Table 4 below shows the comparison of numerical variables between groups (ANOVA), referencing the significant differences found. When comparing myofunctional orofacial characteristics (AMIOFE) in the studied groups, we have found significant differences regarding the total AMIOFE Protocol score $(p=$
$0.0007)$ and postural aspect/condition of the palate $(p=$ 0.0073), where NG differs from GO and GON, being these similar. As for the movement of lips $(p=0.0451)$ and tongue $(p=0.0111)$, the studied groups presented different results among themselves.

Table 4. Comparison of numerical variables among groups (ANOVA)

\begin{tabular}{ccc}
\hline Variable & Factor & p value \\
\hline Age & Group & 0.1550 \\
AMIOFE & & $\mathbf{0 . 0 0 0 7 ^ { 1 }}$ \\
Lips (mobility) & & $\mathbf{0 . 0 4 5 1 ^ { 2 }}$ \\
Tongue (mobility) & & $\mathbf{0 . 0 1 1 1 ^ { 2 }}$ \\
Jaw (mobility) & 0.8099 \\
Cheeks (mobility) & 0.2490 \\
Swallowing & 0.2480 \\
Chewing & 0.1408 \\
\hline Palate & & $\mathbf{0 . 0 0 7 3}^{2}$
\end{tabular}

The Tukey test pointed out the significant differences between the groups where: $1=\mathrm{GN} \neq \mathrm{GO} / \mathrm{GON} ; 2=\mathrm{GN} \neq \mathrm{GO} \neq \mathrm{GON}$. GN = nasal respirator group; $\mathrm{GO}=$ oral respirator group; GON = oronasal respirator group .

In the categorical variable comparative: aspect and posture/position of the lips, there was a significant difference between the studied groups; that is, GN, GO and GON presented different scores for this variable, as shown in Table 5, below. 
Table 5. Comparison of the variable Postural Condition of Lips among the studied groups

\begin{tabular}{ccccc}
\hline $\begin{array}{c}\text { Frequency } \\
\text { Lips }\end{array}$ & RN & RO & RON & Total \\
\hline 3 & 12 & 5 & 8 & 23 \\
& 75.00 & 31.25 & 50.00 & 25 \\
2 & 12 & 11 & 8 & \\
& 25.00 & 68.75 & 0.00 & 0 \\
1 & 0 & 0 & 0.00 & 48 \\
\hline Total & 0.00 & 16 & 16 & 00 \\
\hline
\end{tabular}

Frequency of the categorical variable aspect and postural condition / position of the lips, in Nasal Respirator = RN, Oral Respirator = R0 and Oronasal Respirator $=$ RON with score 3 (normal), 2 (slight change) and 1 (severe change) with significance level of p-value: 0.0456 between groups. (Chi-square test).

The variables evaluated in the AMIOFE protocol were analyzed statistically between RON and RO groups in order to compare if there are more similarities or differences between these two groups and the result pointed to a similarity between them, as seen in Table 6 , below.

Table 6. Quantitative description of the variables evaluated in the AMIOFE protocol between the Oronasal Respirator (RON) and Oral Respirator (R0) groups

\begin{tabular}{|c|c|c|c|c|c|c|c|c|}
\hline Group & Variable & $\mathbf{N}$ & Mean & Median & SD & Min. & Max. & $\mathrm{p}$-value \\
\hline RON & Age & 16 & 7.00 & 6.00 & 1.37 & 6.00 & 10.00 & \\
\hline Ro & Appearance/Posture & 16 & 6.50 & 6.00 & 0.82 & 6.00 & 8.00 & 0.3385 \\
\hline RON & Lips & 16 & 2.56 & 3.00 & 0.51 & 2.00 & 3.00 & \\
\hline RO & Lips & 16 & 2.31 & 2.00 & 0.48 & 2.00 & 3.00 & 0.1673 \\
\hline RON & Jaw & 16 & 2.75 & 3.00 & 0.45 & 2.00 & 3.00 & \\
\hline RO & Jaw & 16 & 2.63 & 3.00 & 0.50 & 2.00 & 3.00 & 0.4670 \\
\hline RON & Cheeks & 16 & 2.06 & 2.00 & 0.57 & 1.00 & 3.00 & \\
\hline RO & Cheeks & 16 & 2.00 & 2.00 & 0.63 & 1.00 & 3.00 & 0.7881 \\
\hline RON & Symmetry & 16 & 1.94 & 2.00 & 0.25 & 1.00 & 2.00 & --- \\
\hline Ro & Symmetry & 16 & 1.94 & 2.00 & 0.25 & 1.00 & 2.00 & --- \\
\hline RON & Tongue & 16 & 2.94 & 3.00 & 0.25 & 2.00 & 3.00 & --- \\
\hline RO & Tongue & 16 & 2.63 & 3.00 & 0.50 & 2.00 & 3.00 & --- \\
\hline RON & Palate & 16 & 2.06 & 2.00 & 0.25 & 2.00 & 3.00 & -- \\
\hline RO & $\begin{array}{l}\text { Palate } \\
\text { Mobility }\end{array}$ & 16 & 1.75 & 2.00 & 0.77 & 1.00 & 3.00 & --- \\
\hline RON & Lips & 16 & 8.63 & 8.50 & 1.71 & 4.00 & 11.00 & \\
\hline RO & Lips & 16 & 8.56 & 8.00 & 1.75 & 6.00 & 12.00 & 0.5631 \\
\hline RON & Tongue & 16 & 15.81 & 16.00 & 1.47 & 12.00 & 18.00 & \\
\hline RO & Tongue & 16 & 16.56 & 16.50 & 1.09 & 15.00 & 18.00 & 0.1896 \\
\hline RON & Jaw & 16 & 12.13 & 12.50 & 2.75 & 5.00 & 15.00 & \\
\hline RO & Jaw & 16 & 12.75 & 13.00 & 1.73 & 9.00 & 15.00 & 0.6742 \\
\hline RON & Cheeks & 16 & 9.25 & 10.00 & 2.35 & 5.00 & 12.00 & \\
\hline RO & $\begin{array}{l}\text { Cheeks } \\
\text { Functions } \\
\text { Breathing }\end{array}$ & 16 & 9.50 & 9.00 & 1.59 & 6.00 & 12.00 & 0.9847 \\
\hline RON & & 16 & 2.00 & 2.00 & 0.00 & 2.00 & 2.00 & --- \\
\hline $\mathrm{RO}$ & & 16 & 1.00 & 1.00 & 0.00 & 1.00 & 1.00 & --- \\
\hline
\end{tabular}




\begin{tabular}{ccccccccc}
\hline Group & Variable & N & Mean & Median & SD & Min. & Max. & p-value \\
\hline RON & Swallowing & & & & & & & \\
RO & & 16 & 9.94 & 10.00 & 1.65 & 6.00 & 12.00 & \\
& & 16 & 8.94 & 9.00 & 1.65 & 6.00 & 13.00 & 0.0551 \\
RON & Chewing & & & & & & & \\
RO & & 16 & 6.25 & 6.00 & 1.48 & 4.00 & 9.00 & \\
RON & & 16 & 6.00 & 6.00 & 1.46 & 4.00 & 9.00 & 0.5509 \\
RO & Total & 16 & 77.94 & 78.50 & 7.19 & 60.00 & 87.00 & \\
\hline
\end{tabular}

Groups studied RON = oronasal respirator and RO = oral respirator; with variables evaluated by AMIOFE; N (sample); Mean and Median; D.P (standard deviation); maximum and minimum values and p-value; --- for variables that could not be measured. With values of significance. The Mann-Whitney test was used to compare the variables. The level of significance adopted for this study was $5 \%$.

\section{DISCUSSION}

In order to define the RON, besides the otorhinolaryngological evaluation, a specific protocol was applied, Orofacial Evaluation with Scores ${ }^{11}$ that allows to define qualitatively and quantitatively different types of breathing.

The age of the patients, 6 to 12 years old, was also determined by the protocol to use only this age group. The average age obtained in the study was \pm 7.21 years old, with no significant difference between $\mathrm{NB}, \mathrm{RO}$ and RON $(p=0.1550)$. These results were also verified in relation to gender, among the groups of $\mathrm{RN}$, $R O, \operatorname{RON}(p=0.07476)$. The data are in agreement with studies in the literature ${ }^{16-18}$ that disregarded the equal distribution of gender in the group. Only the classification of the presence or absence of nasal obstruction was found ${ }^{19-21}$.

Regarding the total AMIOFE score, there is no reference in the published literature on the measurement of the scores of this protocol for the studied population in this study, which obtained median values for the NG groups of $85.5 \%$; GO of $77.5 \%$ and GON of $78.5 \%$.

However, the AMIOFE protocol in this study found a significant difference between the studied groups - RN / RO / RON - $(p=0.0007)$, in agreement with the study that aimed to evaluate myofunctional nasal and oral obese respirators ${ }^{16}$ that also used this same myofunctional assessment protocol and found a significant difference of $p=0.028$ between groups.

The applicability of this protocol to the population for which this research is intended, which is that of children with altered respiratory mode, has found a way to characterize the studied respiratory modes in nasal, oronasal and oral, as already described in a previous study ${ }^{16}$.
Regarding total orofacial myofunctional scores, these were below 80 in the RO and RON groups, thus diagnosing BMD in this population, as in another study ${ }^{16}$.

The variables of appearance and palate posture $(p=$ 0.0073 ) of lips and tongue, besides lip mobility $(p=$ $0.0451)$ and tongue $(p=0.0111)$, showed statistically significant and distinct differences between RO and RON groups, with no significant difference in the other variables between $\mathrm{RO}$ and $\mathrm{RON}$, in this study, however, agreeing with findings in obese individuals ${ }^{16}$.

It was interesting to note that the significant variables found are characteristic of subjects with inefficient lip seal ${ }^{10,22}$. In that the escape of air through the mouth, language lodged in the buccal floor, altered intraoral pressure favors muscle flaccidity and development of a tall and narrow palate ${ }^{23,24}$.

The present study found a mean of 12.13 for the mandibular mobility variable in oronasal, differing from another study ${ }^{25}$, which also used AMOFE in oronasal, which found an average of 14.00 for the same variable.

Another study, involving children with respiratory mode alterations ${ }^{19}$, observed that when individuals presented a respiratory pattern that was farther from normal, there was a greater occurrence of changes in the appearance of the cheeks $(p=0.011)$, which may be related to the decrease in muscle tone.

For this study, the other evaluated variables in AMIOFE did not present a statistically significant difference between RO and RON groups, signaling for the similarity in the orofacial behaviors of these groups.

\section{CONCLUSION}

The results allow us to conclude that the evaluated groups of oral and oronasal breathers presented $\mathrm{BMD}$, as well as the need for attention to the oronasal 
breathing patient, since this group presented similar oromyofunctional characteristics to the oral respirator, suggesting that the early intervention can avoid alterations in the stomatognathic system of these patients.

\section{ACKNOWLEDGEMENTS}

The author thanks Professor Dr. Mírian Hideko Nagae, director of her Master's Dissertation / Unicamp, Professor Dr. Cláudia Maria de Felício and the team of the Laboratory of Research of the Stomatognathic System - LISE, of the Speech Therapy course of the Medical School of Ribeirão Preto, for training for the application of the AMIOFE protocol.

\section{REFERENCES}

1. Degan VV, Puppin-Rontani RM. Aumento da aeração nasal após remoção de hábitos de sucção e terapia miofuncional. Rev. CEFAC. 2007;9(1):55-60.

2. Felício CM, Medeiros AP, Melchior MO. Validity of the protocol of orofacial myofunctional evaluation with scoresnfor young and adult subjects. J Oral Rehabil. 2012;39(10):744-53.

3. Engelke W, Jung $\mathrm{K}$, Knösel $\mathrm{M}$. Intra-oral compartment pressures: a biofunctional model and experimental measurements under different conditions of posture. Clin Oral Investig. 2011;15(2):165-76.

4. Campanha SM, Freire LM, Fontes MJ. Impact of asthma, allergic rhinits and mouth breathing in life quality of children and adolescents. Rev. CEFAC. 2008;10(4):513-9.

5. Migliorucci RR, Passos DCBOF, Berretin-Felix G. Orofacial myofunctional therapy program for individuals undergoing orthognathic surgery. Rev. CEFAC. 2017;19(2):277-88.

6. Rezende MS, Carvalho LC, Prado RAM, Rocha CBJ, Silva VR, Lunes $\mathrm{DH}$. Isostretching method effects on lung function and posture of mouth breathers. ConScientiae Saúde. 2016;15(1):89-95.

7. Brustolin JP, Dalpian DM, Zanatta FBB, Casagrande L. Association between history of breastfeeding and reports of oral habits and allergy in children. Rev. Fac. Odontol. 2012;53(2):11-4.

8. Andrada e Silva MA, Marchesan IQ, Ferreira LP, Schmidt R, Ramires RR. Posture, lips and tongue tone and mobility of mouth breathing children. Rev. CEFAC. 2012;14(5):853-60.
9. Oliveira RLB, Noronha WP, Bonjardim LR. Masticatory performance evaluation in patients with nasal and mouth breathing. Rev. CEFAC. 2012;14(1):114-21.

10. Nagae MH, Alves MC, Kinoshita RL, Bittencourt ZZLC, Gagliardo H. Quality of life in oral and oronasal respirators. Rev. CEFAC. 2013;15(1):105-10.

11. Felício CM, Ferreira CLP. Protocol of orofacial myofunctional evaluation with scores. Int $\mathrm{J}$ Pediatr Otorhinolaryngology. 2008;7(3)367-75.

12. Folha GA. Extension of numerical scales of the Orofacial Evaluation Protocol (AMIOFE), validation and reliability. [Dissertação] Ribeirão Preto (SP): Faculdade de Medicina de Ribeirão Preto - USP; 2010.

13. Felício CM, Da Silva JA. Metodologia psicofísica aplicada à Fonoaudiologia. In: Marchesan IQ, Zorzi JL, Dias Gomes IC (org). Tópicos em fonoaudiologia. São Paulo: Lovise; 1998. p.51-64.

14. Giglio LD. Orofacial biomechanics and masticatory efficiency in young adults. [Dissertação] Ribeirão Preto (SP): Faculdade de Medicina de Ribeirão Preto - USP; 2013.

15. Medeiros APM. Validation of the Orofacial Myofunctional Assessment Protocol with scales for youth and adults. [Dissertação] Ribeirão Preto (SP): Faculdade de Medicina de Ribeirão Preto USP; 2011.

16. Berlese DB. Myofunctional and electromyographic characteristics of the orofacial motricity of obese individuals. [Dissertação] Santa Maria (RS): Universidade Federal de Santa Maria; 2012.

17. Hitos S, Arakaki R, Solé D, Weckx LLM. Oral breathing and speech disorders in children. $J$ Pediatr. 2013;89(4):361-5.

18. Berwig LC, Silva AMT, Côrrea ECR, Moraes AB, Montenegro M, Ritzel RA. Quantitative analysis of the hard palate in different facial typologies in nasal and mouth breathers. Rev. CEFAC. 2012;14(4):616-25.

19. Coêlho JF, França FP, Gaia WO, Almeida LNA, Alves GAS. Correlação entre alterações estruturais e funcionais do sistema estomatognático de crianças e adolescentes respiradores orais. Anais do XXIII Congresso brasileiro e IX Congresso Internacional de Fonoaudiologia-Interdisciplinaridade e Fonoaudiologia, Salvador, Bahia, Brasil. 2015.

20. Ambrosio RA, Trevillatto PC, Sakima T, Ignácio AS, Shimuzu RH. Correlation between morphology and 
function of the upper lip: a longitudinal evaluation. Eur $\mathrm{J}$ of Orthodontics. 2009;31(3):306-13.

21. Dutra EH, Maruo H, Vianna-Lara MS. Eletromyographic activity evaluation and comparison of the orbicularis oris (lower fascicle) and mentalis muscles in predominantly nose- or mouth-breathing subjects. Am J Orthod Dentofacial Orthop. 2006;129(6):722.e1-722.e9.

22. Ricketts RM. The keystone triad. Anatomy, phylogenetics and clinical references. $\mathrm{Am} \mathrm{J}$ Orthodont.1964;5:244-64.

23. Siqueira VCV, Sousa MA, Bérzin F, Casarini CAS. Electromyographic analysis of the orbicularis of the mouth in youngsters with Class II / 1st division, and youngsters with normal occlusion. Dental Press J Orthod. 2011;16(5):54-61.

24. Ung N, Koening J, Shapiro PA, Shapiro G, Trasnk $G$. A quantitative assessment of respiratory patterns and their effects on dentofacial development. Am J Orthod Dentofacial Orthop. 1990;98:523-32.

25. França FP, Coêlho JF, Gaia WO, Almeida LNA, Alves GAS. Estudo eletromiográfico do músculo masseter em crianças e adolescentes respiradores oronasais. Anais do XXIII Congresso brasileiro e IX Congresso Internacional de FonoaudiologiaInterdisciplinaridade e Fonoaudiologia, Salvador, Bahia, Brasil. 2015. 